\title{
Comorbidities and Prescribed Medications in Korean Patients with Chronic Hepatitis C: A Nationwide, Population-Based Study
}

\author{
Jung Wha Chung ${ }^{1}$, Hwa Young Choi ${ }^{2}$, Moran $\mathrm{Ki}^{2}$, Eun Sun Jang ${ }^{1}$, and Sook-Hyang Jeong ${ }^{1}$ \\ ${ }^{1}$ Department of Internal Medicine, Seoul National University Bundang Hospital, Seoul National University College of Medicine, \\ Seongnam, and ${ }^{2}$ National Cancer Center Graduate School of Cancer Science and Policy, Goyang, Korea
}

\author{
Article Info \\ Received November 12, 2019 \\ Revised March 16, 2020 \\ Accepted April 13, 2020 \\ Published online July 6, 2020 \\ Corresponding Author \\ Sook-Hyang Jeong \\ ORCID https://orcid.org/0000-0002-4916-7990 \\ E-mail jsh@snubh.org
}

Jung Wha Chung and Hwa Young Choi contributed equally to this work as first authors.

\begin{abstract}
Background/Aims: Extrahepatic comorbidities and comedication are important to consider in the treatment of chronic hepatitis $\mathrm{C}(\mathrm{CHC})$ patients with direct-acting antivirals (DAAs) due to the risk of drug-drug interaction (DDI) and the effect of comorbidities on clinical outcomes. This study aimed to investigate the detailed profiles of comorbidities and comedication among Korean $\mathrm{CHC}$ patients.

Methods: All adult patients ( $\geq 18$ years old) with a primary diagnostic code of $\mathrm{CHC}$ in 2013 were selected from the National Health Insurance claims database. For each patient, all ICD-10 codes listed as primary or secondary diagnoses and all prescribed medications were collected.

Results: Among 47,104 CHC patients (median age, 57 years; male, 49.3\%), 84.8\% had at least one comorbidity for a mean number of 2.4 , which increased with age. The most prevalent comorbidities were hypertension, esophagitis, dyslipidemia, diabetes mellitus, and peptic ulcer. Overall, $96.8 \%$ of the patients took at least one prescribed medication, with a mean of 8.1 medications/ year, and the three most common drug types were analgesics, gastrointestinal agents, and antibacterials. Use of at least one drug with a DDI risk category of "contraindicated medication" or "required dose-reduction/additional monitoring" was observed in $97 \%$ of the overall patients. The proportion of prescribed medications that were contraindicated with DAAs varied from $2.0 \%$ to $38.9 \%$ depending on the hepatitis $\mathrm{C}$ virus regimen.

Conclusions: The majority of $\mathrm{CHC}$ patients had comorbidities; almost all patients took multiple prescribed medications, the number of which increased with age, and significant DDI risk was present in $97 \%$ of this Korean patient cohort. Comorbidities and comedication profiles should be considered during DAA therapy. (Gut Liver 2021;15:295-306)
\end{abstract}

Key Words: Hepatitis C, chronic; Drug therapy; Drug interactions; Comorbidity; Liver cirrhosis

\section{INTRODUCTION}

Treatment options for chronic hepatitis C virus (HCV) infection have improved with the introduction of directacting antivirals (DAA), resulting in sustained virological response rates of at least $90 \%$ in real-world settings. In contrast to pegylated-interferon- $\alpha$ and ribavirin (RBV) therapy, treatment indications of DAA therapy were widely expanded to include patients with decompensated cirrhosis, renal impairment, organ transplantation recipients under immunosuppressive therapy, and human immunodeficiency virus (HIV) coinfection taking antiretroviral therapy. In general, DAA provides a good option of HCV treatment except for patients with a reduced life expectancy due to extrahepatic comorbidities or progressive hepatocellular carcinoma (HCC). ${ }^{1}$

Extrahepatic comorbidities in HCV patients are important because they could increase morbidity and mortality among chronic hepatitis $\mathrm{C}(\mathrm{CHC})$ patients even after sustained virological response is achieved with DAA therapy. Though DAA can decrease mortality, a recent prospective study demonstrated that 1-year mortality of 1,891 Spanish HCV patients who had undergone DAA treatment was $3.4 \%$, with $70 \%$ of mortalities attributable to extrahepatic 
comorbidities, and the remaining $30 \%$ to hepatic complications. ${ }^{2}$ Therefore, understanding of HCV-related and unrelated extrahepatic comorbidities is essential in the treatment of $\mathrm{CHC}$ patients in DAA era.

Drug-drug interactions (DDIs) can occur whenever a drug affects the activity of another drug by either changing drug concentrations, or through additive, synergistic or antagonistic effects without changing drug concentration. The major mechanism of DDIs are induction or inhibition of drug metabolizing enzymes such as cytochrome P450s, and drug transporters such as P-glycoprotein, multidrug resistance protein MRP2, and organic anion-transporting polypeptides. ${ }^{3}$ One prominent example of DDIs in CHC treatment occurred in 2015, when European and U.S. health agencies issued a safety warning in response to nine cases of severe bradycardia/bradyarrhythmia which occurred after taking ledipasvir/sofosbuvir (LDV/SOF) or SOF in combination with amiodarone and another DAA. Among the nine cases, six developed within 24 hours of DAA initiation, and three required pacemaker implantation, and one mortality occurred. This DDI was replicated in preclinical in vivo experiments using guinea pigs and rhesus monkeys. ${ }^{4}$ Moreover, a case of severe tamoxifen hepatotoxicity was reported which was induced by CYP3A4 interaction with combination therapy of ombitasvir, paritaprevir, ritonavir and dasabuvir $(\mathrm{OBV} / \mathrm{PTV} / \mathrm{r}+\mathrm{DSV}){ }^{5}$

Prevention DDIs and optimal management of extrahepatic comorbid conditions has therefore become a more prominent issue during DAA therapy. However, prevalence of comorbidities and prescription patterns of comedication in CHC patients differ by geographic region and ethnic background. Though these issues are reported in the United States, Japan, and European countries, there is no study in South Korea. Thus, this study aimed to investigate prevalence of comorbidities and to identify the prescribed medication profiles of chronic HCV patients in South Korea using the National Health Insurance (NHI) claims database in 2013.

\section{MATERIALS AND METHODS}

\section{Study design}

The data from the NHI claims database in 2013 were collected retrospectively. $\mathrm{NHI}$ is a government operating obligatory insurance system which covers $97 \%$ of the total population, and claims data are therefore considered to be representative for Korean $\mathrm{HCV}$ patients. The NHI database includes all prescriptions; however, data on medications or procedures that are not covered by insurance such as cosmetic procedures or nutritional supplements, are not avail- able. This study protocol was approved by the Institutional Review Board of the Seoul National University Bundang Hospital (IRB number: X-1704/393-902) and informed consent was waived due to the study using de-identified retrospective health claims.

\section{Patient selection and data retrieval}

All the patients over 18 years of age who had been diagnosed as CHC in 2013 were selected. CHC was defined as International Statistical Classification of Diseases and Related Health Problems 10th Revision (ICD-10) code B18.2 (chronic viral hepatitis $\mathrm{C}$ ) as primary diagnosis. Patients were categorized into four liver diseases group; $\mathrm{CHC}$ (ICD10 code B18.2 without any following ICD-10 codes), liver cirrhosis (LC; ICD-10 codes: K74 [fibrosis and cirrhosis of liver], K74.0 [hepatic fibrosis], K74.1 [hepatic sclerosis], or K74.2 [hepatic fibrosis with hepatic sclerosis]), HCC (ICD-10 codes: C22 [malignant neoplasm of liver and intrahepatic bile ducts], C22.0 [liver cell carcinoma], and C22.9 [malignant neoplasm of liver, not specified as primary or secondary]), and liver transplantation (LT; ICD10 codes: Z94.4 [liver transplant status], or T86.4 [liver transplant failure and rejection]) group. Cirrhotic patients with HCC were categorized as HCC group, and patients in LT group were excluded from the other groups. Therefore, all included patients were classified as $\mathrm{CH}$, LC, HCC, or LT. HCV patients with hepatitis B virus or HIV coinfection were included in the study.

\section{Assessment of the number and types of comorbidities}

Primary and secondary ICD-10 diagnostic codes were captured at every visit in 2013 to classify comorbidities (Supplementary Table 1). Among the ICD-10 codes system, "R codes: Symptoms, signs and abnormal clinical and laboratory findings, not elsewhere classified (Chapter XVIII)" and " $Z$ codes: Factors influencing health status and contact with health services (Chapter XXI)" are nondisease categories and those codes were excluded to avoid bias when comparing the proportion of comorbidities.

Rule-out diagnoses were excluded, and diagnostic codes which were observed at only one visit during a year were excluded, so that only confirmed diagnostic codes observed at least twice in a year were included. HCC (ICD10 codes: C22.0 C22.9) was excluded from the comorbidity category of "Neoplasm," and "sequelae of viral hepatitis" (ICD-10 code: N94.2) were excluded from the comorbidity category of "Certain infectious and parasitic diseases" in order to prevent overestimation of comorbidity numbers.

Proportion of patients with at least one comorbidity and mean number of comorbidities per patient were calculated. 
Moreover, subgroup analyses were conducted according to age, gender, and liver disease diagnostic group, i.e., CHC, LC, HCC, and LT. If a patient had two different ICD-10 codes for a comorbidity belonging to the same category of disease, it was counted as two different comorbidities. For example, if a patient had cardiac arrhythmia and ischemic heart disease, which are both included in the category of "Diseases of the circulatory system," the number of comorbidities for that patient was still considered as being two.

\section{Assessment of the number and types of prescribed medications}

Whole prescription histories of insurance-covered medication for each patient were retrieved and analyzed using specific codes of main substance assigned by Korean Ministry of Health and Welfare. Prescribed medications were classified into therapeutic drug classes based on information derived from "https://www.hep-druginteractions.org/" by Liverpool University (Supplementary Tables 2 and 3). A "Hepatotonics" category was specifically added by investigators because hepatotonics including biphenyldimethyl-dicarboxylate, carnitine orotate, flavin adenine dinucleotide sodium, L-ornithine-L-aspartate, milk thistle (silymarin) or Carduus marianus extract, ursodeoxycholic acid are commonly prescribed medications for $\mathrm{CHC}$ patients in Korea (Supplementary Tables 2 and 3). "Steroids" included injections (i.e., intravenous, intramuscular, subcutaneous, and intra-articular, etc.) or oral administration of glucocorticosteroids, but did not include topical forms of clobetasol, clobetasone, and hydrocortisone, in accordance with the Liverpool University group criteria (https:// www.hep-druginteractions.org/checker).

Mean number of prescribed medications per patient and the proportion of patients taking $\geq 1$ medication were analyzed, and subgroup analyses were conducted stratified by age, gender, and underlying liver disease group. When a patient had been prescribed two or more medications of the same composition with different brand names, or medications with different dosages, the number of prescribed medications was counted as one based on its identical active ingredient even though the drug codes were different. For example, if a patient initially took amlodipine $5 \mathrm{mg}$ for control of high blood pressure and then increased to the 10 $\mathrm{mg}$ dosage after 2 months, the number of medications was counted as 1 , although the drug codes were different.

\section{Assessment of DDls between prescribed co- medications and various DAA regimens against $\mathrm{HCV}$}

After classifying the prescribed medications, predicted interactions between those drugs and therapeutic regimens of DAAs were analyzed. DAAs included for the DDI analy- sis in this study were as below: OBV/PTV/r+DSV, daclatasvir + asunaprevir (DCV/ASV), SOF+DCV, LDV/SOF, $\mathrm{SOF}+\mathrm{RBV}$, and elbasvir/grazoprevir, which were available from 2015 to 2017 in South Korea. Four different DDI categories were created: contraindicated, dose-reduction/ additional monitoring required, no clinically significant interaction expected, and no available information. These four categories were derived from information provided by the Liverpool University and Korean pharmaceutical inserts (https://www.hep-druginteractions.org/).

Patients who were not prescribed any medications in 2013 were excluded in this analysis. Patients who were prescribed $\geq 2$ medications that were expected to have two different interaction categories were considered to be exposed to greater DDI interactions. For example, if a patient was prescribed three medications and each of them was categorized as "contraindicated," "dose-reduction/additional monitoring required," and "no available information" with a certain HCV regimen, the patient was considered as having a DDI category of "contraindicated" medications. Additionally, if a patient were prescribed medications with "no available information" and "no clinically significant interaction expected" with a certain HCV regimen, respectively, he was considered as having a risk of DDI with "no available information."

\section{Statistical analysis}

In parametric analyses the minimum and maximum are presented to the same number of decimal places as the

Table 1. Baseline Demographics and Diagnostic Categories of Patients with Chronic Hepatitis C Virus Infection

\begin{tabular}{lc}
\hline \multicolumn{1}{c}{ Variable } & Value $(\mathrm{n}=47,104)$ \\
\hline Age, yr & $57(48-67)$ \\
$18-34$ & $2,219(4.7)$ \\
$35-44$ & $5,650(12.0)$ \\
$45-54$ & $11,767(25.0)$ \\
$55-64$ & $12,577(26.7)$ \\
$65-74$ & $10,233(21.7)$ \\
$\geq 75$ & $4,658(9.9)$ \\
Sex & \\
Male & $23,202(49.3)$ \\
Female & $23,902(50.7)$ \\
Diagnosis of liver disease* & \\
Chronic hepatitis C & $38,850(82.5)$ \\
Liver cirrhosis & $5,882(12.5)$ \\
Hepatocellular carcinoma & $2,271(4.8)$ \\
Post-liver transplantation status & $101(0.2)$ \\
\hline
\end{tabular}

Data are presented as median (interquartile range) or number (\%). *Diagnosis of liver disease was classified by the hospital claim data, and patients with hepatocellular carcinoma or liver transplantation were excluded from the liver cirrhosis group despite having the corresponding diagnostic codes. 
original data. The mean, median, lower and upper quartiles were presented to one more decimal place than the original data. In summary tables of categorical variables, numbers and proportions were used. All statistical analyses were performed using SAS version 9.2 (SAS Institute Inc., Cary, NC, USA) or higher for Windows.

\section{RESULTS}

\section{Demographic characteristics of HCV patients in 2013}

A total of 47,104 patients were identified having the primary diagnostic code of $\mathrm{CHC}$ in 2013 , with a median age of 57.4 years and almost half (49.3\%) being male (Table 1). Patients over 45 years of age accounted for $83.3 \%$ of the total. The proportion of the patients with LC, HCC, and LT were $12.5 \%, 4.8 \%$, and $0.2 \%$, respectively. As severity of liver disease increased, the proportion of old aged patients $\geq 65$ years increased: $28.1 \%(\mathrm{n}=10,932)$ in chronic hepatitis group, $46.3 \%(n=2,721)$ in LC group, and $53.3 \%(n=1,211)$ in HCC group were over age 65 (data not shown).

\section{Comorbidity profiles: types and number of the comorbidities among 47,104 HCV patients}

Overall, $84.8 \%$ of patients had one or more comorbidities, and comorbidity number increased with age, to the extent that $93.6 \%$ of patients $\geq 65$ years old had more than one comorbidity (Fig. 1A). The mean number of comorbidities also increased with age, with the 18 to 34 years having an average of 1.0 comorbidity each, and the $\geq 65$ years group having an average of 3.2 comorbidities each (Fig. 1B). Compared to males, female patients showed slightly higher prevalence of comorbidities across all ages. Top three most prevalent comorbidity categories higher than $50 \%$ among patients were circulatory diseases, (52.8\%), endocrine, nutritional and metabolic diseases (52.4\%), and digestive diseases (50.0\%). Then, mental and behavioral disorders (25.6\%), and nervous diseases followed (Table 2).

The five most common diseases accompanying HCV infected patients were hypertension (31.8\%), esophagitis or gastroesophageal reflux disease (30.0\%), dyslipidemia (21.2\%), diabetes mellitus (20.0\%), and peptic ulcer or gastrointestinal ulcer (18.3\%) (Supplementary Table 4). The prevalence of hypertension and diabetes mellitus was as high as $59.6 \%$ and $29.7 \%$, respectively, especially in the patients $\geq 75$ years. Patients with hepatitis B virus coinfection were founded in $4.3 \%$ (Supplementary Table 4 ). The prevalence of the five most common categories of comorbidity was higher in the LC group than in the $\mathrm{CHC}$ group, and higher in the HCC group than in the LC group (Supplementary Tables 5, 6). Prevalence of circulatory diseases increased with age in all of the $\mathrm{CHC}, \mathrm{LC}$, and HCC groups (Supplementary Fig. 1).
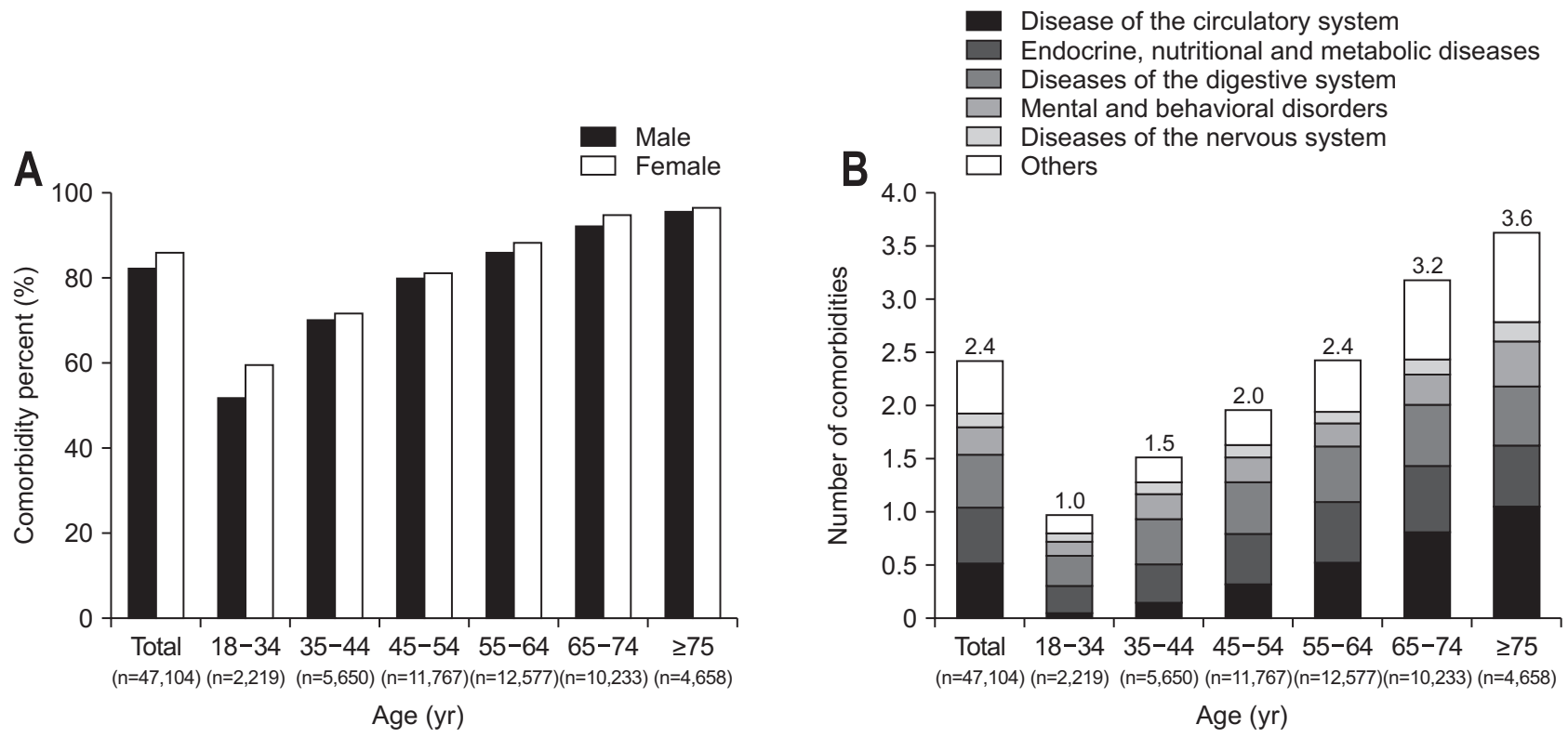

Fig. 1. Comorbidities, defined by International Statistical Classification of Diseases and Related Health Problems 10th Revision (ICD-10) codes, according to age group in Korean chronic hepatitis $C$ patients $(n=47,104)$; (A) the proportion of patients having at least one comorbidity and (B) the mean number of comorbidities per patient. Hepatocellular carcinoma (ICD-10 codes: C22, C22.0 and C22.9) was excluded from the comorbidity category of "Neoplasm." Acute hepatitis (ICD-10 codes: B15-17) and sequelae of viral hepatitis (ICD-10 code: N94.2) were excluded from the comorbidity category of "Certain infectious and parasitic diseases" in order to prevent overestimation of viral hepatitis. 
Table 2. Number and Proportion of Chronic Hepatitis C Patients with At Least One Comorbidity According to Age and Sex

\begin{tabular}{|c|c|c|c|c|c|c|c|c|c|}
\hline \multirow[b]{2}{*}{ Comorbidity (ICD-10 codes) } & \multirow{2}{*}{$\begin{array}{c}\text { Total } \\
(n=47,104)\end{array}$} & \multicolumn{6}{|c|}{ Age group of chronic hepatitis C patients, yr } & \multicolumn{2}{|c|}{ Sex } \\
\hline & & $\begin{array}{c}18-34 \\
(n=2,219)\end{array}$ & $\begin{array}{c}35-44 \\
(n=5,650)\end{array}$ & $\begin{array}{c}45-54 \\
(n=11,767)\end{array}$ & $\begin{array}{c}55-64 \\
(n=12,577)\end{array}$ & $\begin{array}{c}65-74 \\
(n=10,233)\end{array}$ & $\begin{array}{c}\geq 75 \\
(n=4,658)\end{array}$ & $\begin{array}{c}\text { Male } \\
(n=23,202)\end{array}$ & $\begin{array}{c}\text { Female } \\
(n=23,902)\end{array}$ \\
\hline $\begin{array}{l}\text { Diseases of the circulatory system } \\
\text { (100-199) }\end{array}$ & $\begin{array}{l}24,889 \\
(52.8)\end{array}$ & $\begin{array}{l}132 \\
(5.9)\end{array}$ & $\begin{array}{c}880 \\
(15.6)\end{array}$ & $\begin{array}{l}3,861 \\
(32.8)\end{array}$ & $\begin{array}{l}6,707 \\
(53.3)\end{array}$ & $\begin{array}{l}8,362 \\
(81.7)\end{array}$ & $\begin{array}{c}4,947 \\
(106.2)\end{array}$ & $\begin{array}{l}12,184 \\
(52.5)\end{array}$ & $\begin{array}{l}12,705 \\
(53.2)\end{array}$ \\
\hline $\begin{array}{l}\text { Endocrine, nutritional and meta- } \\
\text { bolic diseases (E00-E90) }\end{array}$ & $\begin{array}{l}24,676 \\
(52.4)\end{array}$ & $\begin{array}{c}567 \\
(25.6)\end{array}$ & $\begin{array}{l}2,051 \\
(36.3)\end{array}$ & $\begin{array}{l}5,627 \\
(47.8)\end{array}$ & $\begin{array}{l}7,250 \\
(57.6)\end{array}$ & $\begin{array}{l}6,502 \\
(63.5)\end{array}$ & $\begin{array}{l}2,679 \\
(57.5)\end{array}$ & $\begin{array}{l}11,432 \\
(49.3)\end{array}$ & $\begin{array}{l}13,244 \\
(55.4)\end{array}$ \\
\hline $\begin{array}{l}\text { Diseases of the digestive system } \\
\text { (K00-K93) }\end{array}$ & $\begin{array}{l}23,542 \\
(50.0)\end{array}$ & $\begin{array}{c}616 \\
(27.8)\end{array}$ & $\begin{array}{l}2,422 \\
(42.9)\end{array}$ & $\begin{array}{l}5,730 \\
(48.7)\end{array}$ & $\begin{array}{l}6,476 \\
(51.5)\end{array}$ & $\begin{array}{l}5,732 \\
(56.0)\end{array}$ & $\begin{array}{l}2,566 \\
(55.1)\end{array}$ & $\begin{array}{l}11,689 \\
(50.4)\end{array}$ & $\begin{array}{l}11,853 \\
(49.6)\end{array}$ \\
\hline $\begin{array}{l}\text { Mental and behavioral disorders } \\
\text { (F00-F99) }\end{array}$ & $\begin{array}{l}12,045 \\
(25.6)\end{array}$ & $\begin{array}{c}303 \\
(13.7)\end{array}$ & $\begin{array}{l}1,305 \\
(23.1)\end{array}$ & $\begin{array}{l}2,771 \\
(23.5)\end{array}$ & $\begin{array}{l}2,782 \\
(22.1)\end{array}$ & $\begin{array}{l}2,933 \\
(28.7)\end{array}$ & $\begin{array}{l}1,951 \\
(41.9)\end{array}$ & $\begin{array}{l}5,109 \\
(22.0)\end{array}$ & $\begin{array}{l}6,936 \\
(29.0)\end{array}$ \\
\hline $\begin{array}{l}\text { Diseases of the nervous system } \\
\text { (G00-G99] }\end{array}$ & $\begin{array}{l}5,875 \\
(12.5)\end{array}$ & $\begin{array}{l}169 \\
(7.6)\end{array}$ & $\begin{array}{c}644 \\
(11.4)\end{array}$ & $\begin{array}{l}1,265 \\
(10.8)\end{array}$ & $\begin{array}{l}1,359 \\
(10.8)\end{array}$ & $\begin{array}{l}1,538 \\
(15.0)\end{array}$ & $\begin{array}{c}900 \\
(19.3)\end{array}$ & $\begin{array}{l}2,567 \\
(11.1)\end{array}$ & $\begin{array}{l}3,308 \\
(13.8)\end{array}$ \\
\hline $\begin{array}{l}\text { Diseases of the musculoskeletal } \\
\text { system and connective tissue } \\
\text { (M00-M99) }\end{array}$ & $\begin{array}{l}5,683 \\
(12.1)\end{array}$ & $\begin{array}{c}34 \\
(1.5)\end{array}$ & $\begin{array}{c}144 \\
(2.5)\end{array}$ & $\begin{array}{l}702 \\
(6.0)\end{array}$ & $\begin{array}{l}1,462 \\
(11.6)\end{array}$ & $\begin{array}{l}2,221 \\
(21.7)\end{array}$ & $\begin{array}{l}1,120 \\
(24.0)\end{array}$ & $\begin{array}{l}889 \\
(3.8)\end{array}$ & $\begin{array}{l}4,794 \\
(20.1)\end{array}$ \\
\hline $\begin{array}{l}\text { Diseases of the respiratory system } \\
\text { (J00-J99) }\end{array}$ & $\begin{array}{l}5,041 \\
(10.7)\end{array}$ & $\begin{array}{l}109 \\
(4.9)\end{array}$ & $\begin{array}{l}313 \\
(5.5)\end{array}$ & $\begin{array}{l}860 \\
(7.3)\end{array}$ & $\begin{array}{l}1,271 \\
(10.1)\end{array}$ & $\begin{array}{l}1,629 \\
(15.9)\end{array}$ & $\begin{array}{c}859 \\
(18.4)\end{array}$ & $\begin{array}{l}2,369 \\
(10.2)\end{array}$ & $\begin{array}{l}2,672 \\
(11.2)\end{array}$ \\
\hline $\begin{array}{l}\text { Diseases of the eye and adnexa } \\
\text { (H00-H59) }\end{array}$ & $\begin{array}{r}4,519 \\
(9.6)\end{array}$ & $\begin{array}{c}31 \\
(1.4)\end{array}$ & $\begin{array}{c}174 \\
(3.1)\end{array}$ & $\begin{array}{l}652 \\
(5.5)\end{array}$ & $\begin{array}{l}1,180 \\
(9.4)\end{array}$ & $\begin{array}{l}1,641 \\
(16.0)\end{array}$ & $\begin{array}{c}841 \\
(18.1)\end{array}$ & $\begin{array}{c}2,178 \\
(9.4)\end{array}$ & $\begin{array}{c}2,341 \\
(9.8)\end{array}$ \\
\hline Neoplasms (COO-D48)* & $\begin{array}{l}3,306 \\
(7.0)\end{array}$ & $\begin{array}{c}23 \\
(1.0)\end{array}$ & $\begin{array}{l}166 \\
(2.9)\end{array}$ & $\begin{array}{l}593 \\
(5.0)\end{array}$ & $\begin{array}{c}953 \\
(7.6)\end{array}$ & $\begin{array}{l}1,026 \\
(10.0)\end{array}$ & $\begin{array}{c}545 \\
(11.7)\end{array}$ & $\begin{array}{l}1,611 \\
(6.9)\end{array}$ & $\begin{array}{l}1,695 \\
(7.1)\end{array}$ \\
\hline $\begin{array}{l}\text { Certain infectious and parasitic dis- } \\
\text { eases (A00-B99) (including HBV, } \\
\text { HIV, and Tb) }\end{array}$ & $\begin{array}{c}2,613 \\
(5.5)\end{array}$ & $\begin{array}{l}139 \\
(6.3)\end{array}$ & $\begin{array}{l}336 \\
(5.9)\end{array}$ & $\begin{array}{l}712 \\
(6.1)\end{array}$ & $\begin{array}{l}703 \\
(5.6)\end{array}$ & $\begin{array}{l}502 \\
(4.9)\end{array}$ & $\begin{array}{l}221 \\
(4.7)\end{array}$ & $\begin{array}{l}1,504 \\
(6.5)\end{array}$ & $\begin{array}{l}1,109 \\
(4.6)\end{array}$ \\
\hline $\begin{array}{l}\text { Diseases of the genitourinary sys- } \\
\text { tem (N00-N99) }\end{array}$ & $\begin{array}{l}1,311 \\
(2.8)\end{array}$ & $\begin{array}{c}25 \\
(1.1)\end{array}$ & $\begin{array}{c}107 \\
(1.9)\end{array}$ & $\begin{array}{l}256 \\
(2.2)\end{array}$ & $\begin{array}{l}334 \\
(2.7)\end{array}$ & $\begin{array}{l}356 \\
(3.5)\end{array}$ & $\begin{array}{l}233 \\
(5.0)\end{array}$ & $\begin{array}{l}781 \\
(3.4)\end{array}$ & $\begin{array}{l}530 \\
(2.2)\end{array}$ \\
\hline $\begin{array}{l}\text { Diseases of the skin and subcuta- } \\
\text { neous tissue (L00-L99) }\end{array}$ & $\begin{array}{c}572 \\
(1.2)\end{array}$ & $\begin{array}{c}18 \\
(0.8)\end{array}$ & $\begin{array}{c}72 \\
(1.3)\end{array}$ & $\begin{array}{l}148 \\
(1.3)\end{array}$ & $\begin{array}{c}134 \\
(1.1)\end{array}$ & $\begin{array}{c}136 \\
(1.3)\end{array}$ & $\begin{array}{c}64 \\
(1.4)\end{array}$ & $\begin{array}{l}367 \\
(1.6)\end{array}$ & $\begin{array}{c}205 \\
(0.9)\end{array}$ \\
\hline $\begin{array}{l}\text { Symptoms, signs and abnormal } \\
\text { clinical and laboratory findings, not } \\
\text { elsewhere classified (R00-R99) }\end{array}$ & $\begin{array}{c}147 \\
(0.3)\end{array}$ & $\begin{array}{c}6 \\
(0.3)\end{array}$ & $\begin{array}{c}17 \\
(0.3)\end{array}$ & $\begin{array}{c}34 \\
(0.3)\end{array}$ & $\begin{array}{c}32 \\
(0.3)\end{array}$ & $\begin{array}{c}40 \\
(0.4)\end{array}$ & $\begin{array}{c}18 \\
(0.4)\end{array}$ & $\begin{array}{c}89 \\
(0.4)\end{array}$ & $\begin{array}{c}58 \\
(0.2)\end{array}$ \\
\hline $\begin{array}{l}\text { Diseases of the blood and blood- } \\
\text { forming organs and certain } \\
\text { disorders involving the immune } \\
\text { mechanism (D50-D89) }\end{array}$ & $\begin{array}{c}5 \\
{[<0.1)}\end{array}$ & 0 & 0 & 0 & $\begin{array}{c}2 \\
{[<0.1\}}\end{array}$ & $\begin{array}{c}3 \\
{[<0.1)}\end{array}$ & 0 & $\begin{array}{c}1 \\
\{<0.1\}\end{array}$ & $\begin{array}{c}4 \\
{[<0.1\}}\end{array}$ \\
\hline
\end{tabular}

Data are presented as number $(\%)$.

ICD-10, International Statistical Classification of Diseases and Related Health Problems 10th Revision; HBV, hepatitis B virus; HIV, human immunodeficiency virus; Tb, tuberculosis.

*Hepatocellular carcinoma (ICD-10: C22, C22.0 and C22.9) was excluded from the comorbidity category of "Neoplasm"; ${ }^{\dagger}$ Acute hepatitis (ICD-10: B15-17), chronic hepatitis C (ICD-10: B18.2), and sequelae of viral hepatitis (ICD-10: B94.2) were excluded from the comorbidity category of "Certain infectious and parasitic diseases."

\section{Proportion and mean number of the prescribed medications among 47,104 HCV patients}

A total of 379,536 medications were prescribed to the $47,104 \mathrm{CHC}$ patients in a year and the mean number of prescriptions was 8.1 per patient in 2013 (Table 3). Overall, $96.8 \%$ of patients took at least one prescribed medication: $88.4 \%$ of patients in the 18 to 34 years of age group and $99.0 \%$ of patients in the $\geq 75$ years group (Supplementary Fig. 2). Patients in the 18 to 34 years of age group were prescribed an average of 5.4 medications, but as the number of medications increased with age, patients in the $\geq 75$ years group were prescribed an average of 9.8 medications (Fig. 2). Use of at least one drug having the DDIs risk cat- egory of "contraindicated medication" and "required dosereduction/additional monitoring" was present in 55.5\% and $41.3 \%$ of the overall patients, respectively (Fig. 3), and the risk proportion increased with age (Fig. 4). The most common classes of prescribed medications were analgesics $(83.3 \%)$, gastrointestinal agents $(80.1 \%)$, antibacterials (67.9\%), "anticoagulant, antiplatelet and fibrinolytic" (59.1\%) and oral and injection forms of steroids (52.7\%), in order (Table 3).

Females were generally prescribed more medications than male patients except for the $\geq 75$ years of age group. Compared to female patients, male patients were more frequently prescribed hepatotonics ( $55.7 \%$ vs $47.6 \%$ ), herb- 
Table 3. Comparison of the Number and Proportion of Prescribed Medications in Each Drug Classification According to Age and Sex in Korean Chronic Hepatitis C Patients

\begin{tabular}{|c|c|c|c|c|c|c|c|c|c|}
\hline \multirow{2}{*}{$\begin{array}{l}\text { Classification of } \\
\text { prescribed medication* }\end{array}$} & \multirow{2}{*}{$\begin{array}{c}\text { Total } \\
(n=47,104)\end{array}$} & \multicolumn{6}{|c|}{ Age group of chronic hepatitis $C$ patients, yr } & \multicolumn{2}{|c|}{ Sex } \\
\hline & & $\begin{array}{c}18-34 \\
(n=2,219)\end{array}$ & $\begin{array}{c}35-44 \\
(n=5,650)\end{array}$ & $\begin{array}{c}45-54 \\
(n=11,767)\end{array}$ & $\begin{array}{c}55-64 \\
(n=12,577)\end{array}$ & $\begin{array}{c}65-74 \\
(n=10,233)\end{array}$ & $\begin{array}{c}\geq 75 \\
(n=4,658)\end{array}$ & $\begin{array}{c}\text { Male } \\
(n=23,202)\end{array}$ & $\begin{array}{c}\text { Female } \\
\text { (n=23,902) }\end{array}$ \\
\hline Analgesics & $\begin{array}{l}39,225 \\
(83.3)\end{array}$ & $\begin{array}{l}1,526 \\
(68.8)\end{array}$ & $\begin{array}{l}4,330 \\
(76.6)\end{array}$ & $\begin{array}{l}9,427 \\
(80.1)\end{array}$ & $\begin{array}{l}10,565 \\
(84.0)\end{array}$ & $\begin{array}{l}9,139 \\
(89.3)\end{array}$ & $\begin{array}{l}4,238 \\
(91.0)\end{array}$ & $\begin{array}{l}18,628 \\
(80.3)\end{array}$ & $\begin{array}{l}20,597 \\
(86.2)\end{array}$ \\
\hline Gastrointestinal agents & $\begin{array}{l}37,715 \\
(80.1)\end{array}$ & $\begin{array}{l}1,440 \\
(64.9)\end{array}$ & $\begin{array}{l}4,183 \\
(74.0)\end{array}$ & $\begin{array}{l}9,099 \\
(77.3)\end{array}$ & $\begin{array}{l}10,173 \\
(80.9)\end{array}$ & $\begin{array}{l}8,748 \\
(85.5)\end{array}$ & $\begin{array}{l}4,072 \\
(87.4)\end{array}$ & $\begin{array}{l}17,725 \\
(76.4)\end{array}$ & $\begin{array}{l}19,990 \\
(83.6)\end{array}$ \\
\hline Antibacterials & $\begin{array}{l}31,993 \\
(67.9)\end{array}$ & $\begin{array}{l}1,476 \\
(66.5)\end{array}$ & $\begin{array}{l}3,939 \\
(69.7)\end{array}$ & $\begin{array}{l}7,760 \\
(65.9)\end{array}$ & $\begin{array}{l}8,406 \\
(66.8)\end{array}$ & $\begin{array}{l}7,141 \\
(69.8)\end{array}$ & $\begin{array}{l}3,271 \\
(70.2)\end{array}$ & $\begin{array}{l}14,908 \\
(64.3)\end{array}$ & $\begin{array}{l}17,085 \\
(71.5)\end{array}$ \\
\hline $\begin{array}{l}\text { Anticoagulant, antiplatelet } \\
\text { and fibrinolytic }\end{array}$ & $\begin{array}{l}27,842 \\
(59.1)\end{array}$ & $\begin{array}{l}1,106 \\
(49.8)\end{array}$ & $\begin{array}{l}3,054 \\
(54.1)\end{array}$ & $\begin{array}{l}6,637 \\
(56.4)\end{array}$ & $\begin{array}{l}7,485 \\
(59.5)\end{array}$ & $\begin{array}{l}6,619 \\
(64.7)\end{array}$ & $\begin{array}{l}2,941 \\
(63.1)\end{array}$ & $\begin{array}{l}13,096 \\
(56.4)\end{array}$ & $\begin{array}{l}14,746 \\
(61.7)\end{array}$ \\
\hline Steroids $^{\dagger}$ & $\begin{array}{c}24,802 \\
(52.7)\end{array}$ & $\begin{array}{c}900 \\
(40.6)\end{array}$ & $\begin{array}{l}2,517 \\
(44.5)\end{array}$ & $\begin{array}{l}5,791 \\
(49.2)\end{array}$ & $\begin{array}{l}6,725 \\
(53.5)\end{array}$ & $\begin{array}{l}6,127 \\
(59.9)\end{array}$ & $\begin{array}{l}2,742 \\
(58.9)\end{array}$ & $\begin{array}{l}11,370 \\
(49.0)\end{array}$ & $\begin{array}{l}13,432 \\
(56.2)\end{array}$ \\
\hline Hepatotonics ${ }^{\ddagger}$ & $\begin{array}{l}24,303 \\
(51.6)\end{array}$ & $\begin{array}{c}565 \\
(25.5)\end{array}$ & $\begin{array}{l}2,280 \\
(40.4)\end{array}$ & $\begin{array}{l}5,662 \\
(48.1)\end{array}$ & $\begin{array}{l}6,334 \\
(50.4)\end{array}$ & $\begin{array}{l}6,181 \\
(60.4)\end{array}$ & $\begin{array}{l}3,281 \\
(70.4)\end{array}$ & $\begin{array}{l}12,931 \\
(55.7)\end{array}$ & $\begin{array}{l}11,372 \\
(47.6)\end{array}$ \\
\hline $\begin{array}{l}\text { Anxiolytics/hypnotics/ } \\
\text { sedatives }\end{array}$ & $\begin{array}{l}21,583 \\
(45.8)\end{array}$ & $\begin{array}{c}501 \\
(22.6)\end{array}$ & $\begin{array}{l}2,162 \\
(38.3)\end{array}$ & $\begin{array}{l}5,068 \\
(43.1)\end{array}$ & $\begin{array}{l}5,732 \\
(45.6)\end{array}$ & $\begin{array}{l}5,466 \\
(53.4)\end{array}$ & $\begin{array}{l}2,654 \\
(57.0)\end{array}$ & $\begin{array}{l}9,635 \\
(41.5)\end{array}$ & $\begin{array}{l}11,948 \\
(50.0)\end{array}$ \\
\hline $\begin{array}{l}\text { Herbals/supplements/ } \\
\text { vitamins }^{\S}\end{array}$ & $\begin{array}{l}18,765 \\
(39.8)\end{array}$ & $\begin{array}{c}431 \\
(19.4)\end{array}$ & $\begin{array}{l}1,639 \\
(29.0)\end{array}$ & $\begin{array}{l}4,377 \\
(37.2)\end{array}$ & $\begin{array}{l}4,926 \\
(39.2)\end{array}$ & $\begin{array}{l}4,826 \\
(47.2)\end{array}$ & $\begin{array}{l}2,566 \\
(55.1)\end{array}$ & $\begin{array}{l}9,601 \\
(41.4)\end{array}$ & $\begin{array}{l}9,164 \\
(38.3)\end{array}$ \\
\hline Antihistamines & $\begin{array}{l}17,912 \\
(38.0)\end{array}$ & $\begin{array}{c}758 \\
(34.2)\end{array}$ & $\begin{array}{l}2,126 \\
(37.6)\end{array}$ & $\begin{array}{l}4,204 \\
(35.7)\end{array}$ & $\begin{array}{l}4,723 \\
(37.6)\end{array}$ & $\begin{array}{l}4,230 \\
(41.3)\end{array}$ & $\begin{array}{l}1,871 \\
(40.2)\end{array}$ & $\begin{array}{l}8,189 \\
(35.3)\end{array}$ & $\begin{array}{l}9,723 \\
(40.7)\end{array}$ \\
\hline $\begin{array}{l}\text { Hypertension/heart failure } \\
\text { agents }\end{array}$ & $\begin{array}{l}15,035 \\
(31.9)\end{array}$ & $\begin{array}{c}81 \\
(3.7)\end{array}$ & $\begin{array}{c}643 \\
(11.4)\end{array}$ & $\begin{array}{l}2,678 \\
(22.8)\end{array}$ & $\begin{array}{l}4,063 \\
(32.3)\end{array}$ & $\begin{array}{l}4,800 \\
(46.9)\end{array}$ & $\begin{array}{l}2,770 \\
(59.5)\end{array}$ & $\begin{array}{l}7,307 \\
(31.5)\end{array}$ & $\begin{array}{l}7,728 \\
(32.3)\end{array}$ \\
\hline Antiarrhythmics & $\begin{array}{l}12,926 \\
(27.4)\end{array}$ & $\begin{array}{c}264 \\
(11.9)\end{array}$ & $\begin{array}{l}1,044 \\
(18.5)\end{array}$ & $\begin{array}{l}2,784 \\
(23.7)\end{array}$ & $\begin{array}{l}3,505 \\
(27.9)\end{array}$ & $\begin{array}{l}3,580 \\
(35.0)\end{array}$ & $\begin{array}{l}1,749 \\
(37.5)\end{array}$ & $\begin{array}{l}5,815 \\
(25.1)\end{array}$ & $\begin{array}{l}7,111 \\
(29.8)\end{array}$ \\
\hline Calcium channel blockers & $\begin{array}{l}10,505 \\
(22.3)\end{array}$ & $\begin{array}{c}43 \\
(1.9)\end{array}$ & $\begin{array}{l}384 \\
(6.8)\end{array}$ & $\begin{array}{l}1,728 \\
(14.7)\end{array}$ & $\begin{array}{l}2,831 \\
(22.5)\end{array}$ & $\begin{array}{l}3,459 \\
(33.8)\end{array}$ & $\begin{array}{l}2,060 \\
(44.2)\end{array}$ & $\begin{array}{l}5,159 \\
(22.2)\end{array}$ & $\begin{array}{l}5,346 \\
(22.4)\end{array}$ \\
\hline Antidepressants & $\begin{array}{l}8,155 \\
(17.3)\end{array}$ & $\begin{array}{l}167 \\
(7.5)\end{array}$ & $\begin{array}{c}819 \\
(14.5)\end{array}$ & $\begin{array}{l}1,938 \\
(16.5)\end{array}$ & $\begin{array}{l}2,068 \\
(16.4)\end{array}$ & $\begin{array}{l}2,076 \\
(20.3)\end{array}$ & $\begin{array}{l}1,087 \\
(23.3)\end{array}$ & $\begin{array}{l}3,513 \\
(15.1)\end{array}$ & $\begin{array}{l}4,642 \\
(19.4)\end{array}$ \\
\hline Antidiabetics & $\begin{array}{l}7,722 \\
(16.4)\end{array}$ & $\begin{array}{c}36 \\
(1.6)\end{array}$ & $\begin{array}{r}418 \\
(7.4)\end{array}$ & $\begin{array}{l}1,695 \\
(14.4)\end{array}$ & $\begin{array}{l}2,169 \\
(17.2)\end{array}$ & $\begin{array}{l}2,310 \\
(22.6)\end{array}$ & $\begin{array}{l}1,094 \\
(23.5)\end{array}$ & $\begin{array}{l}4,492 \\
(19.4)\end{array}$ & $\begin{array}{l}3,230 \\
(13.5)\end{array}$ \\
\hline Lipid lowering agents & $\begin{array}{l}7,452 \\
(15.8)\end{array}$ & $\begin{array}{c}62 \\
(2.8)\end{array}$ & $\begin{array}{l}428 \\
(7.6)\end{array}$ & $\begin{array}{l}1,538 \\
(13.1)\end{array}$ & $\begin{array}{l}2,362 \\
(18.8)\end{array}$ & $\begin{array}{l}2,166 \\
(21.2)\end{array}$ & $\begin{array}{c}896 \\
(19.2)\end{array}$ & $\begin{array}{l}3,414 \\
(14.7)\end{array}$ & $\begin{array}{l}4,038 \\
(16.9)\end{array}$ \\
\hline Hepatitis drugs" & $\begin{array}{l}7,405 \\
(15.7)\end{array}$ & $\begin{array}{c}335 \\
(15.1)\end{array}$ & $\begin{array}{l}1,198 \\
(21.2)\end{array}$ & $\begin{array}{l}2,486 \\
(21.1)\end{array}$ & $\begin{array}{l}2,157 \\
(17.2)\end{array}$ & $\begin{array}{l}1,067 \\
(10.4)\end{array}$ & $\begin{array}{l}162 \\
(3.5)\end{array}$ & $\begin{array}{l}3,787 \\
(16.3)\end{array}$ & $\begin{array}{l}3,618 \\
(15.1)\end{array}$ \\
\hline $\begin{array}{l}\text { Anesthetics and muscle } \\
\text { relaxants }\end{array}$ & $\begin{array}{l}5,261 \\
(11.2)\end{array}$ & $\begin{array}{l}168 \\
(7.6)\end{array}$ & $\begin{array}{l}537 \\
(9.5)\end{array}$ & $\begin{array}{l}1,240 \\
(10.5)\end{array}$ & $\begin{array}{l}1,387 \\
(11.0)\end{array}$ & $\begin{array}{l}1,341 \\
(13.1)\end{array}$ & $\begin{array}{c}588 \\
(12.6)\end{array}$ & $\begin{array}{l}2,454 \\
(10.6)\end{array}$ & $\begin{array}{l}2,807 \\
(11.7)\end{array}$ \\
\hline Anticonvulsants & $\begin{array}{l}4,904 \\
(10.4)\end{array}$ & $\begin{array}{c}73 \\
(3.3)\end{array}$ & $\begin{array}{l}419 \\
(7.4)\end{array}$ & $\begin{array}{l}1,085 \\
(9.2)\end{array}$ & $\begin{array}{l}1,228 \\
(9.8)\end{array}$ & $\begin{array}{l}1,356 \\
(13.3)\end{array}$ & $\begin{array}{c}743 \\
(16.0)\end{array}$ & $\begin{array}{l}2,392 \\
(10.3)\end{array}$ & $\begin{array}{l}2,512 \\
(10.5)\end{array}$ \\
\hline Bronchodilators & $\begin{array}{l}4,723 \\
(10.0)\end{array}$ & $\begin{array}{l}176 \\
(7.9)\end{array}$ & $\begin{array}{l}498 \\
(8.8)\end{array}$ & $\begin{array}{l}952 \\
(8.1)\end{array}$ & $\begin{array}{l}1,264 \\
(10.1)\end{array}$ & $\begin{array}{l}1,222 \\
(11.9)\end{array}$ & $\begin{array}{c}611 \\
(13.1)\end{array}$ & $\begin{array}{l}2,113 \\
(9.1)\end{array}$ & $\begin{array}{l}2,610 \\
(10.9)\end{array}$ \\
\hline Antifungals & $\begin{array}{c}4,474 \\
(9.5)\end{array}$ & $\begin{array}{c}259 \\
(11.7)\end{array}$ & $\begin{array}{c}627 \\
(11.1)\end{array}$ & $\begin{array}{l}1,181 \\
(10.0)\end{array}$ & $\begin{array}{l}1,095 \\
(8.7)\end{array}$ & $\begin{array}{l}904 \\
(8.8)\end{array}$ & $\begin{array}{l}408 \\
(8.8)\end{array}$ & $\begin{array}{r}2,051 \\
(8.8)\end{array}$ & $\begin{array}{l}2,423 \\
(10.1)\end{array}$ \\
\hline Parkinsonism agents & $\begin{array}{l}3,809 \\
(8.1)\end{array}$ & $\begin{array}{l}104 \\
(4.7)\end{array}$ & $\begin{array}{l}349 \\
(6.2)\end{array}$ & $\begin{array}{l}874 \\
(7.4)\end{array}$ & $\begin{array}{l}1,043 \\
(8.3)\end{array}$ & $\begin{array}{l}978 \\
(9.6)\end{array}$ & $\begin{array}{l}461 \\
(9.9)\end{array}$ & $\begin{array}{l}1,714 \\
(7.4)\end{array}$ & $\begin{array}{l}2,095 \\
(8.8)\end{array}$ \\
\hline Beta blockers & $\begin{array}{c}2,998 \\
(6.4)\end{array}$ & $\begin{array}{c}23 \\
(1.0)\end{array}$ & $\begin{array}{c}108 \\
(1.9)\end{array}$ & $\begin{array}{l}463 \\
(3.9)\end{array}$ & $\begin{array}{c}767 \\
(6.1)\end{array}$ & $\begin{array}{c}1,013 \\
(9.9)\end{array}$ & $\begin{array}{c}624 \\
(13.4)\end{array}$ & $\begin{array}{c}1,467 \\
(6.3)\end{array}$ & $\begin{array}{c}1,531 \\
(6.4)\end{array}$ \\
\hline Bisphosphonates & $\begin{array}{c}2,741 \\
(5.8)\end{array}$ & $\begin{array}{c}4 \\
(0.2)\end{array}$ & $\begin{array}{c}14 \\
(0.2)\end{array}$ & $\begin{array}{c}132 \\
(1.1)\end{array}$ & $\begin{array}{l}590 \\
(4.7)\end{array}$ & $\begin{array}{l}1,248 \\
(12.2)\end{array}$ & $\begin{array}{c}753 \\
(16.2)\end{array}$ & $\begin{array}{l}244 \\
(1.1)\end{array}$ & $\begin{array}{l}2,497 \\
(10.4)\end{array}$ \\
\hline $\begin{array}{r}\text { Antipsychotics/ } \\
\text { neuroleptics }\end{array}$ & $\begin{array}{l}2,099 \\
(4.5)\end{array}$ & $\begin{array}{c}54 \\
(2.4)\end{array}$ & $\begin{array}{l}322 \\
(5.7)\end{array}$ & $\begin{array}{l}636 \\
(5.4)\end{array}$ & $\begin{array}{l}451 \\
(3.6)\end{array}$ & $\begin{array}{l}357 \\
(3.5)\end{array}$ & $\begin{array}{l}279 \\
(6.0)\end{array}$ & $\begin{array}{l}1,187 \\
(5.1)\end{array}$ & $\begin{array}{l}912 \\
(3.8)\end{array}$ \\
\hline Antiprotozoals & $\begin{array}{l}1,690 \\
(3.6)\end{array}$ & $\begin{array}{l}148 \\
(6.7)\end{array}$ & $\begin{array}{l}242 \\
(4.3)\end{array}$ & $\begin{array}{l}427 \\
(3.6)\end{array}$ & $\begin{array}{l}418 \\
(3.3)\end{array}$ & $\begin{array}{l}327 \\
(3.2)\end{array}$ & $\begin{array}{l}128 \\
(2.7)\end{array}$ & $\begin{array}{l}644 \\
(2.8)\end{array}$ & $\begin{array}{l}1,046 \\
(4.4)\end{array}$ \\
\hline Cytotoxics & $\begin{array}{l}987 \\
(2.1)\end{array}$ & $\begin{array}{c}11 \\
(0.5)\end{array}$ & $\begin{array}{c}39 \\
(0.7)\end{array}$ & $\begin{array}{l}172 \\
(1.5)\end{array}$ & $\begin{array}{l}260 \\
(2.1)\end{array}$ & $\begin{array}{l}330 \\
(3.2)\end{array}$ & $\begin{array}{l}175 \\
(3.8)\end{array}$ & $\begin{array}{l}482 \\
(2.1)\end{array}$ & $\begin{array}{l}505 \\
(2.1)\end{array}$ \\
\hline Oxytocics & $\begin{array}{l}968 \\
(2.1)\end{array}$ & $\begin{array}{c}63 \\
(2.8)\end{array}$ & $\begin{array}{c}96 \\
(1.7)\end{array}$ & $\begin{array}{l}223 \\
(1.9)\end{array}$ & $\begin{array}{l}251 \\
(2.0)\end{array}$ & $\begin{array}{l}229 \\
(2.2)\end{array}$ & $\begin{array}{l}106 \\
(2.3)\end{array}$ & $\begin{array}{l}424 \\
(1.8)\end{array}$ & $\begin{array}{l}544 \\
(2.3)\end{array}$ \\
\hline Antivirals & $\begin{array}{l}661 \\
(1.4)\end{array}$ & $\begin{array}{c}35 \\
(1.6)\end{array}$ & $\begin{array}{c}57 \\
(1.0)\end{array}$ & $\begin{array}{l}143 \\
(1.2)\end{array}$ & $\begin{array}{l}169 \\
(1.3)\end{array}$ & $\begin{array}{c}164 \\
(1.6)\end{array}$ & $\begin{array}{c}93 \\
(2.0)\end{array}$ & $\begin{array}{l}248 \\
(1.1)\end{array}$ & $\begin{array}{l}413 \\
(1.7)\end{array}$ \\
\hline
\end{tabular}


Table 3. Continued

\begin{tabular}{|c|c|c|c|c|c|c|c|c|c|}
\hline \multirow{2}{*}{$\begin{array}{l}\text { Classification of } \\
\text { prescribed medication* }\end{array}$} & \multirow{2}{*}{$\begin{array}{c}\text { Total } \\
(n=47,104)\end{array}$} & \multicolumn{6}{|c|}{ Age group of chronic hepatitis $C$ patients, $y r$} & \multicolumn{2}{|c|}{ Sex } \\
\hline & & $\begin{array}{c}18-34 \\
(n=2,219)\end{array}$ & $\begin{array}{c}35-44 \\
(n=5,650)\end{array}$ & $\begin{array}{c}45-54 \\
(n=11,767)\end{array}$ & $\begin{array}{c}55-64 \\
\text { (n=12,577) }\end{array}$ & $\begin{array}{c}65-74 \\
(n=10,233)\end{array}$ & $\begin{array}{c}\geq 75 \\
(n=4,658)\end{array}$ & $\begin{array}{c}\text { Male } \\
(n=23,202)\end{array}$ & $\begin{array}{c}\text { Female } \\
(n=23,902)\end{array}$ \\
\hline Antimigraine agents & $\begin{array}{l}644 \\
(1.4)\end{array}$ & $\begin{array}{c}76 \\
(3.4)\end{array}$ & $\begin{array}{r}110 \\
(1.9)\end{array}$ & $\begin{array}{l}162 \\
(1.4)\end{array}$ & $\begin{array}{l}151 \\
(1.2)\end{array}$ & $\begin{array}{c}98 \\
(1.0)\end{array}$ & $\begin{array}{c}47 \\
(1.0)\end{array}$ & $\begin{array}{l}169 \\
(0.7)\end{array}$ & $\begin{array}{l}475 \\
(2.0)\end{array}$ \\
\hline Immunosuppressants & $\begin{array}{l}232 \\
(0.5)\end{array}$ & $\begin{array}{c}5 \\
(0.2)\end{array}$ & $\begin{array}{c}23 \\
(0.4)\end{array}$ & $\begin{array}{c}65 \\
(0.6)\end{array}$ & $\begin{array}{c}83 \\
(0.7)\end{array}$ & $\begin{array}{c}49 \\
(0.5)\end{array}$ & $\begin{array}{c}7 \\
(0.2)\end{array}$ & $\begin{array}{l}126 \\
(0.5)\end{array}$ & $\begin{array}{l}106 \\
(0.4)\end{array}$ \\
\hline Anthelmintics & $\begin{array}{c}45 \\
(0.1)\end{array}$ & 0 & $\begin{array}{c}2 \\
(<0.1)\end{array}$ & $\begin{array}{c}12 \\
(0.1)\end{array}$ & $\begin{array}{c}13 \\
(0.1)\end{array}$ & $\begin{array}{c}11 \\
(0.1)\end{array}$ & $\begin{array}{c}7 \\
(0.2)\end{array}$ & $\begin{array}{c}36 \\
(0.2)\end{array}$ & $\begin{array}{c}9 \\
(<0.1)\end{array}$ \\
\hline $\begin{array}{l}\text { Human immunodeficiency } \\
\text { virus drugs }\end{array}$ & $\begin{array}{c}35 \\
(0.1)\end{array}$ & $\begin{array}{c}3 \\
(0.1)\end{array}$ & $\begin{array}{c}13 \\
(0.2)\end{array}$ & $\begin{array}{c}10 \\
(0.1)\end{array}$ & $\begin{array}{c}6 \\
(<0.1)\end{array}$ & $\begin{array}{c}2 \\
(<0.1)\end{array}$ & $\begin{array}{c}1 \\
\{<0.1)\end{array}$ & $\begin{array}{c}30 \\
(0.1)\end{array}$ & $\begin{array}{c}5 \\
(<0.1)\end{array}$ \\
\hline Other drugs" & $\begin{array}{c}29,924 \\
(63.5)\end{array}$ & $\begin{array}{l}1,046 \\
(47.1)\end{array}$ & $\begin{array}{l}3,170 \\
(56.1)\end{array}$ & $\begin{array}{l}6,951 \\
(59.1)\end{array}$ & $\begin{array}{l}8,140 \\
(64.7)\end{array}$ & $\begin{array}{l}7,268 \\
(71.0)\end{array}$ & $\begin{array}{l}3,349 \\
(71.9)\end{array}$ & $\begin{array}{l}14,657 \\
(63.2)\end{array}$ & $\begin{array}{l}15,267 \\
(63.9)\end{array}$ \\
\hline $\begin{array}{l}\text { Total number of prescrip- } \\
\text { tion (per person) }\end{array}$ & $\begin{array}{c}379,535 \\
(8.05)\end{array}$ & $\begin{array}{l}11,939 \\
(5.38)\end{array}$ & $\begin{array}{c}37,790 \\
(6.68)\end{array}$ & $\begin{array}{l}87,600 \\
(7.44)\end{array}$ & $\begin{array}{c}101,540 \\
(8.07)\end{array}$ & $\begin{array}{c}94,832 \\
(9.26)\end{array}$ & $\begin{array}{l}45,834 \\
(9.84)\end{array}$ & $\begin{array}{c}180,008 \\
(7.75)\end{array}$ & $\begin{array}{c}199,527 \\
(8.34)\end{array}$ \\
\hline
\end{tabular}

Data are presented as number (\%).

*Prescribed drugs were classified based on the drug interaction information provided online by Liverpool University (https://www.hep-druginteractions.org/checker) and Peking University (http://newywxhzy.ashermed.com). A complete list of drugs in each category is shown in Supplementary Table 3; 'The category of "steroids" was defined as injected (i.e., intravenous, intramuscular, subcutaneous, intra-articular, etc.) or orally administered steroids and excluded topical clobetasol, clobetasone, and hydrocortisone based on the drug interaction information provided online by

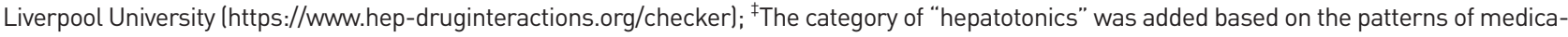
tion consumption in Korea; biphenyl-dimethyl-dicarboxylate, carnitine orotate, flavin adenine dinucleotide sodium, L-ornithine-L-aspartate, milk thistle (silymarin) or Carduus marianus extract, and ursodeoxycholic acid were included (Supplementary Table 3); ${ }^{\S}$ The "herbals/supplements/ vitamins" category of the Liverpool list was not properly assessed in Korea because these drugs are mostly "non-insurance coverage medications," which are not included in the National Health Insurance database; "The category of "hepatitis drugs" included medications for treating chronic hepatitis B (e.g., entecavir, tenofovir, etc.) or chronic hepatitis C (e.g., sofosbuvir, ledipasvir, etc.; Supplementary Table 3); "The category of "other drugs" included acamprosate, alfuzosin, allopurinol, cilostazol, dutasteride, levothyroxine, minoxidil, modafinil, potassium, tamsulosin, etc. (Supplementary Table 3); "The total number of prescriptions was divided by the number of people in each group. A total of 379,535 medications were prescribed to chronic hepatitis $\mathrm{C}$ patients in a year, and the mean number of prescriptions per person was 8.05.

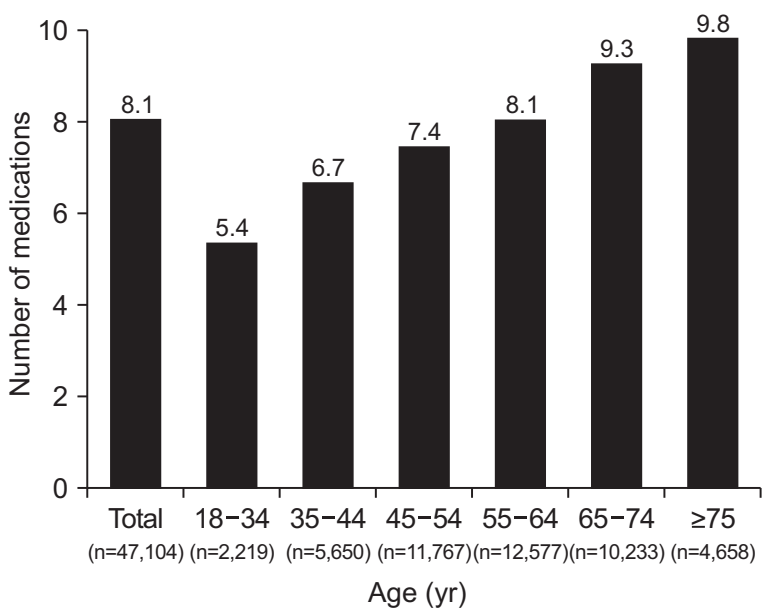

Fig. 2. The mean number of prescription drugs per patient according to age group in Korean chronic hepatitis C patients ( $n=47,104)$. Prescribed medications were classified into therapeutic drug classes based on information derived from https://www.hep-druginteractions. org/ by Liverpool University (Supplementary Table 2). The "hepatotonics" category was specifically listed by the investigators because hepatotonics are one of the most commonly prescribed classes of medication in Korea.

als/supplements/vitamins (41.4\% vs $38.3 \%)$, antidiabetics (19.4\% vs $13.5 \%)$, hepatitis drugs ( $16.3 \%$ vs $15.1 \%)$ and antipsychotics/neuroleptics ( $5.1 \%$ vs $3.8 \%)$. However, analge- sics, gastrointestinal agents, antibacterials, "anticoagulant, antiplatelet and fibrinolytic," and steroids were prescribed more often in female than in male.

\section{Predicted DDls between prescribed co-medications and various DAA regimens against $\mathrm{HCV}$}

The potential risk of the four types of predicted DDIs according to each HCV regimen are presented in Fig. 3. The proportion of contraindicated medications were observed in $2.0 \%$ with elbasvir/grazoprevir, $2.3 \%$ with SOF+RBV, $4.2 \%$ with LDV/SOF, $27.4 \%$ with SOF/DCV, $38.7 \%$ with DCV+ASV and $38.9 \%$ with OBV/PTV/ r+DSV. Among genotype 1 regimen, compared to elbasvir/ grazoprevir, OBV/PTV/r+DSV (odds ratio [OR], 19.01; 95\% confidence interval [CI], 17.82 to 20.30), DCV+ASV (OR, 18.9; 95\% CI, 17.72 to 20.19), SOF+DCV (OR, 13.4; 95\% CI, 12.55 to 14.32 ), and LDV/SOF (OR, 2.05; 95\% CI, 1.89 to 2.21 ) showed a significantly higher proportion of contraindicated medication number (all $\mathrm{p}<0.0001$ ). Among genotype 2 regimen, compared to SOF/RBV, LDV/ SOF (OR, 1.79; 95\% CI, 1.66 to 1.93 ), and SOF/DCV (OR, 11.72; 95\% CI, 11.02 to 12.47 ) showed a significantly higher proportion of contraindicated medication number as well (all $\mathrm{p}<0.0001)$. 


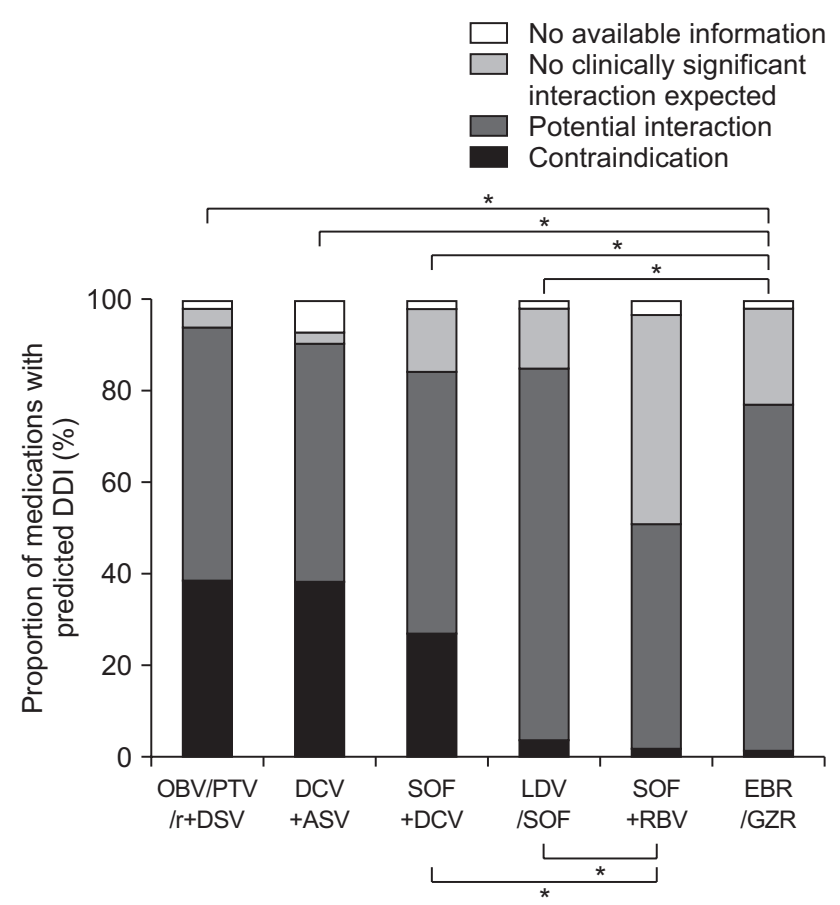

Therapeutic regimen

Fig. 3. The proportions of the types of predicted drug-drug interaction between the prescribed medications and each direct-acting antiviral regimen available in Korean chronic hepatitis $C$ patients $(n=47,104)$. The predicted proportions of contraindicated medications for each regimen are presented with statistical analysis. Among the genotype 1 regimens, compared to EBR/GZR, OBV/PTV/r+DSV lodds ratio [OR], 19.01; $95 \%$ confidence interval [CI], 17.82 to 20.30 ), DCV+ASV (OR, $18.9 ; 95 \% \mathrm{Cl}, 17.72$ to 20.19 ), SOF+DCV (OR, $13.4 ; 95 \% \mathrm{Cl}, 12.55$ to 14.32), and LDV/SOF (OR, 2.05; $95 \% \mathrm{Cl}, 1.89$ to 2.21) showed significantly increased proportions of contraindicated medications. Among the genotype 2 regimen, compared to SOF/RBV, LDV/SOF (OR, 1.79; $95 \% \mathrm{Cl}, 1.66$ to 1.93 ), and SOF/DCV (OR, $11.72 ; 95 \% \mathrm{Cl}, 11.02$ to 12.47 ) showed a significantly increased proportions of contraindicated medications as well lall $* p<0.0001$ ). The predicted interactions are based on the information derived from https://www.hep-druginteractions. org/ by Liverpool University and from the Korean pharmaceutical inserts.

OBV, ombitasvir; PTV, paritaprevir; r, ritonavir; DSV, dasabuvir; DCV, daclatasvir; ASV, asunaprevir; SOF, sofosbuvir; LDV, ledipasvir; RBV, ribavirin; EBR, elbasvir; GZR, grazoprevir; DDI, drug-drug interaction.

The whole list of prescribed medications with significant DDIs were presented in Table 4 and Supplementary Table 7. Steroids (12,221 prescriptions, $25.9 \%)$, gastrointestinal agents (6,993 prescriptions, 14.8\%), and lipid lowering agents (6,822 prescriptions, $14.5 \%)$ were the three most commonly prescribed medication classes. As an individual drug, dexamethasone which has a potential risk of significant DDI with DCV+ASV or SOF+DCV, was the most commonly prescribed medications (12,221 prescription cases, $25.9 \%)$, followed by domperidone (6,993 cases, $14.8 \%$ ) which has significant DDI with OBV/PTV/r+DSV. Moreover, prescription numbers of drugs having predicted

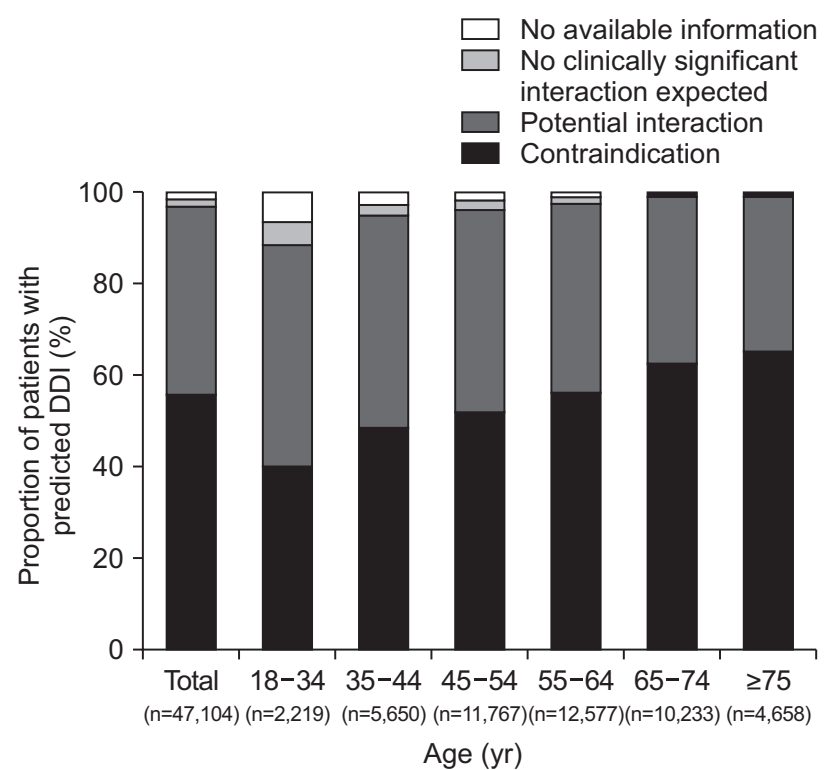

Fig. 4. The proportions of Korean chronic hepatitis $C$ patients $(n=47,104)$ who were prescribed at least one drug with a drug-drug interaction (DDI) risk according to age group. Use of at least one drug in the DDI risk category of "contraindicated medication" increased with age. The predicted interactions are based on the information derived from https://www.hep-druginteractions.org/ by Liverpool University and from the Korean pharmaceutical inserts.

significant DDI risk with all six DAA regimens were as follows: carbamazepine (416 prescriptions), rifampicin (270 prescriptions), phenobarbital (205 prescriptions), oxcarbazepine (47 prescriptions), primidone (6 prescriptions), and phenytoin (3 prescriptions).

\section{DISCUSSION}

Among 47,104 patients with primary diagnosis of $\mathrm{CHC}$ in South Korea, $85 \%$ had at least one comorbid diagnostic code with an average number of 2.4 comorbidities, which increased with age. The five most prevalent comorbidities were hypertension, esophagitis (or gastroesophageal reflux disease), dyslipidemia, diabetes mellitus, and peptic ulcer (or gastrointestinal ulcer). Almost all HCV patients were prescribed at least one medication, with the average number being 8.1 medications per year. The three most commonly prescribed drug classes were analgesics, gastrointestinal agents, and antibacterials. The potential risk of DDIs with category of "contraindicated medication" or "required dose-reduction/additional monitoring" was present in 97\% of the overall patients. The proportion of the prescribed medications that are contraindicated with DAAs varied from $2.0 \%$ to $38.9 \%$ depending on the different $\mathrm{HCV}$ regimen.

$\mathrm{HCV}$ infection is associated with extrahepatic mani- 


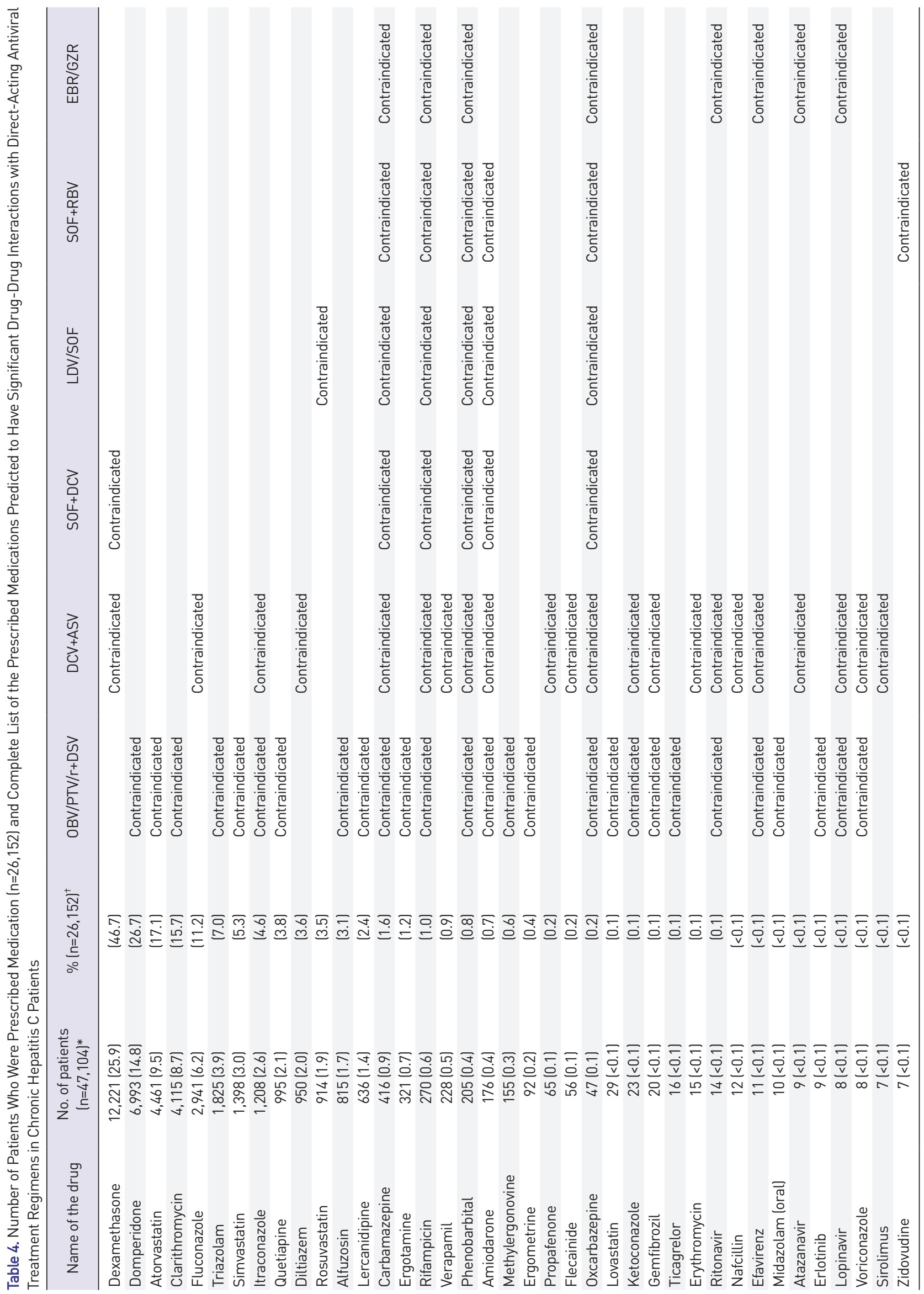




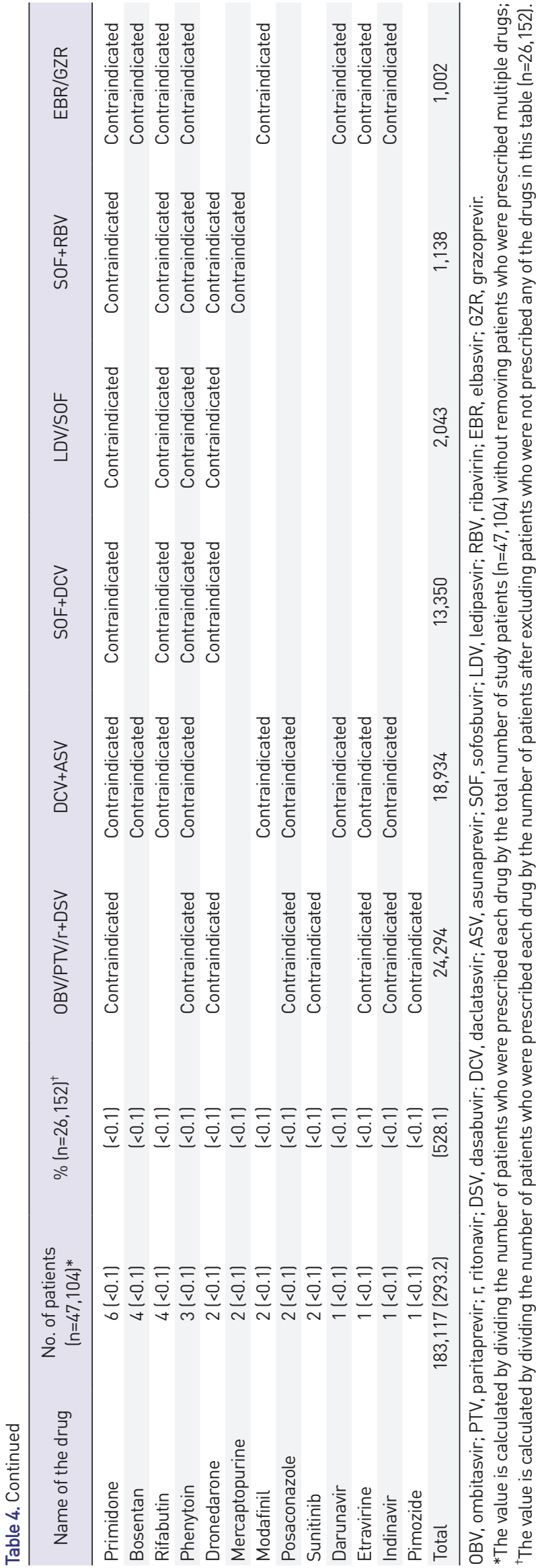

festations or comorbidity, which impact the quality of life and increasing non-liver related mortality rate. The mechanisms causing extrahepatic effect of $\mathrm{HCV}$ infection is not clear, but are likely multifactorial including due to the impact of HCV on the endocrine system, HCV replication in extrahepatic cells, or a heightened immune reaction with systemic effects. ${ }^{6-8}$ Successful eradication of HCV has been shown to improve some of these extrahepatic effects, such as resolution of cryoglobulinemia, reduced insulin resistance, reduced incidence of diabetes and stroke, and frequency of fatigue. ${ }^{6,9}$ A Swedish nationwide populationbased register study ( $n=34,633$ in 2013, mean age of 49 years, $64 \%$ male) showed that $41.3 \%$ of CHC patients had at least one Charlson comorbidity with higher prevalence of cardiovascular, cerebrovascular, and peripheral vascular diseases, chronic pulmonary disease, diabetes, peptic ulcer disease, renal disease and psychiatric disorders compared to matched controls in the general population. ${ }^{5}$

In this nationwide Korean study, $85 \%$ of $\mathrm{CHC}$ patients had at least one comorbidity showing a different pattern of comorbidities between sex. In female patients, musculoskeletal and connective diseases are more common than in males, while infectious diseases including hepatitis B virus, $\mathrm{HIV}$, and tuberculosis, and genitourinary diseases being higher in male CHC patients. In a study using Japanese hospital-based medical claims database $(n=128,967$, median age of 70 years),${ }^{10} 70.5 \%$ of $\mathrm{CHC}$ patients had at least one comorbidity during 23 months, and the most common comorbidities were gastrointestinal diseases $(41.7 \%)$, followed by hypertensive diseases (31.4\%), metabolic disorders (28.2\%) and diabetes mellitus (26.1\%). Similar to the high prevalence found in our study, a study using a U.S. medical claims database for 2 years $(n=7,411$, median age of 49 years) ${ }^{11}$ showed that almost all HCV patients (99.4\%) had at least one comorbidity, including essential hypertension (32.6\%), disorders of lipid metabolism (25.9\%), and gastrointestinal disorders (24.4\%). In a study including 6,278 CHC patients from 59 U.K. specialist centers ${ }^{12}$ (median age of 52 years, $59.1 \%$ of patients had acquired HCV through intravenous drug use), the most common comorbidities were depression (26.1\%), diabetes (11.3\%), nonhepatic malignancy (5.0\%), and HIV coinfection (5.0\%). Though comorbidity profiles differ across countries, common patterns were observed: comorbidity numbers increased with age, and the most common comorbidities are cardiovascular, metabolic and gastrointestinal diseases in the $\mathrm{CHC}$ patients.

For co-medications, the three most commonly prescribed medications were analgesics $(83.3 \%)$, gastrointestinal agents (80.1\%), and antibacterials (67.9\%) among the Korean CHC patients, in comparison to a study in Japan 
reporting that proton pump inhibitors (14.0\%), calcium antagonists (12.5\%) and angiotensin-II antagonists (9.0\%) were most common. ${ }^{10}$ The difference could be explained by the difference in definition of comedication according to the study, for example, co-medications were defined as at least one prescription per year in our study, in comparison to being defined as at least 180 prescription days in the Japanese study. Our definition was based on our desire to account for potential short-term as well as long-term administration of medications that could influence DAA therapy, and demonstrate the large number of prescribed medications in CHC patients (mean 8.1 medications from 5.4 to 9.8 medications through age group), because duration of DAA therapy is short, mostly for 8 to 12 weeks.

The number of antibacterial prescription was 31,993 in this study, which was remarkably high. Although we did not know the exact reason, it reflected a prescription pattern in South Korea. In addition, the drug counting method of our study may relate to the high number of prescription. If a patient is admitted for bacterial pneumonia treated with intravenous injection of ceftriaxone and oral azithromycin, after treating with amoxicillin at an outpatient clinic the number of prescribed antibacterials is three during the one period of pneumonia.

In this study, the potential risk of the four types of predicted DDIs according to each HCV regimen was demonstrated. Moreover, 39 contraindicated medications identified among 47,104 prescriptions in the real-world setting of Korea. The proportion of contraindicated medications was observed as $2.0 \%$ to $39 \%$ depending on the DAA regimen. Therefore, regimens showing less proportion of contraindicated medication may be favorable for the doctors and their patients especially having multiple medications.

Among medications having a potential DDI with DAA therapy, dexamethasone, clarithromycin, fluconazole, and alfuzosin are commonly prescribed medications for "diseases of musculoskeletal system and connective tissue," "certain infectious and parasitic diseases" and "diseases of the genitourinary system." The unexpected high prescription rate of dexamethasone (25.9\% of the CHC patients) in Korea may reflect the high prevalence of intra-articular or muscular injection procedures for musculoskeletal illness, especially in female patients, reflecting the high proportion of musculoskeletal degenerative diseases in older aged people among $\mathrm{CHC}$ patients. Therefore, general physicians or physicians from non-hepatology department who tend to prescribe these steroids or other contraindicated medications should pay more attention when patients are on DAA therapy for $\mathrm{CHC}$. Also, hepatologist should be aware that such medications are commonly prescribed in the other clinics for $\mathrm{CHC}$ patients, and pay more attention to DDIs when choosing DAA regimen for hepatitis $\mathrm{C}$ patients.

Meanwhile, HIV coinfection was only found in 28 patients $(0.06 \%)$ in Korea, which is much lower than in Western countries. ${ }^{10,11}$ Tuberculosis was identified in 201 patients, which led to consideration of DDI, especially rifampicin, one of the contraindicated drugs for all of six DAA regimens. In a U.K. study including a high proportion of intravenous drug users, ${ }^{12}$ the most common medications with DDI potential were psychotropic agents (antidepressants, opioids, and hypnotics) (38.6\%), antidiabetics (9.3\%), immunosuppressants (6.1\%), statins (4.9\%), and antiretrovirals (4.9\%). Therefore, different profiles of comedication and DDI should be considered in the different countries or races.

Additional pharmacokinetic considerations are required for the patients with advanced stage liver disease. HCV infection itself has been shown to impair drug metabolism by reducing microsomal enzymatic activity ${ }^{3}$ and CYP enzyme activity is impaired as liver disease progresses. ${ }^{4}$ Furthermore, CYP activity could be altered by with increased age. ${ }^{6}$ Moreover, since liver disease severity is associated with age, ${ }^{13}$ aged HCV patients with advanced liver diseases accompanying multiple comorbidities and co-medications are particularly vulnerable to the DDI risk. In addition, unascertained mechanisms may contribute to DDI and therefore predictions may not always reflect the clinical situation.

This is the first Korean study to systemically describe comorbidities and prescribed medications in CHC patients using a large nationally-representative database. However, there are several limitations in this study. Because of the nature of administrative data, errors or omissions of diagnostic codes could exist. All prescribed medication codes were collected, but patients may not have taken all prescribed medications. Over-the-counter drugs were not included in the claims database, thus, practical co-medications in the real world might be underestimated. The risk for DDI with dietary intake and other health supplements were not determined. Moreover, DDI with glecaprevir/ pibrentasvir regimen was not included because it was approved after this study completion. In addition, because of complexity of data, we did not compare the data in HCV patients with a non-HCV population. Nevertheless, patients' baseline characteristics in this study are very similar to that of the recent prospective multicenter cohort study in Korea ${ }^{14}$ supporting that this data are representative of Korean CHC patients. Indeed, this study can provide physicians with information about comorbidities and comedications that might be taken into consideration when treating $\mathrm{CHC}$ patients.

In conclusion, majority of the $\mathrm{CHC}$ patients had comor- 
bidities, almost all the patients took multiple prescribed medications per year, which were linearly increasing with age, and significant DDI risk was present in $97 \%$ of the Korean patients. Consideration of comorbidity and comedication profile should be emphasized during DAA therapy.

\section{CONFLICTS OF INTEREST}

No potential conflict of interest relevant to this article was reported.

\section{ACKNOWLEDGEMENTS}

This study was supported by a grant of Gilead (40200 1171), and a grant for the Chronic Infectious Disease Cohort Study (Korea HCV Cohort Study, 4800-4859-304) from the Korea Centers for Disease Control and Prevention.

\section{AUTHOR CONTRIBUTIONS}

Study conception: S.H.J. Study design and revision: S.H.J., M.K., E.S.J. Data collection: H.Y.C. Data analysis: J.W.C., H.Y.C., S.H.J. Manuscript drafting: J.W.C., S.H.J. All authors read and approved the final manuscript.

\section{ORCID}

Jung Wha Chung https://orcid.org/0000-0003-3648-3406 Hwa Young Choi https://orcid.org/0000-0002-8195-9250 Moran Ki https://orcid.org/0000-0002-8892-7104 Eun Sun Jang https://orcid.org/0000-0003-4274-2582 Sook-Hyang Jeong

https://orcid.org/0000-0002-4916-7990

\section{REFERENCES}

1. Smolders EJ, Ter Horst PJG, Wolters S, Burger DM. Cardiovascular risk management and hepatitis $\mathrm{C}$ : combining drugs.
Clin Pharmacokinet 2019;58:565-592.

2. Smolders EJ, Berden FA, de Kanter CT, Kievit W, Drenth JP, Burger DM. The majority of hepatitis $\mathrm{C}$ patients treated with direct acting antivirals are at risk for relevant drug-drug interactions. United European Gastroenterol J 2017;5:648-657.

3. Herold C, Heinz R, Niedobitek G, Schneider T, Hahn EG, Schuppan D. Quantitative testing of liver function in relation to fibrosis in patients with chronic hepatitis B and C. Liver 2001;21:260-265

4. Frye RF, Zgheib NK, Matzke GR, et al. Liver disease selectively modulates cytochrome P450: mediated metabolism. Clin Pharmacol Ther 2006;80:235-245.

5. Wyffels K, Horsmans Y. Tamoxifen-induced hepatotoxicity caused by drug interaction with direct-acting antiviral agents for hepatitis C. J Oncol Pharm Pract 2019;25:2038-2040.

6. Bebia Z, Buch SC, Wilson JW, et al. Bioequivalence revisited: influence of age and sex on CYP enzymes. Clin Pharmacol Ther 2004;76:618-627.

7. Tang L, Marcell L, Kottilil S. Systemic manifestations of hepatitis C infection. Infect Agent Cancer 2016;11:29.

8. Zignego AL, Gragnani L, Piluso A, et al. Virus-driven autoimmunity and lymphoproliferation: the example of $\mathrm{HCV}$ infection. Expert Rev Clin Immunol 2015;11:15-31.

9. Pradat P, Voirin N, Tillmann HL, Chevallier M, Trépo C. Progression to cirrhosis in hepatitis $\mathrm{C}$ patients: an agedependent process. Liver Int 2007;27:335-339.

10. Ruzicka DJ, Tetsuka J, Fujimoto G, Kanto T. Comorbidities and co-medications in populations with and without chronic hepatitis C virus infection in Japan between 2015 and 2016. BMC Infect Dis 2018;18:237.

11. Louie KS, St Laurent S, Forssen UM, Mundy LM, Pimenta JM. The high comorbidity burden of the hepatitis $C$ virus infected population in the United States. BMC Infect Dis 2012;12:86.

12. Hudson B, Walker AJ, Irving WL. Comorbidities and medications of patients with chronic hepatitis $\mathrm{C}$ under specialist care in the UK. J Med Virol 2017;89:2158-2164.

13. Massard J, Ratziu V, Thabut D, et al. Natural history and predictors of disease severity in chronic hepatitis C. J Hepatol 2006;44:S19-S24.

14. Nam JY, Jang ES, Kim YS, et al. Epidemiological and clinical characteristics of hepatitis C virus infection in South Korea from 2007 to 2017: a prospective multicenter cohort study. Gut Liver 2020;14:207-217. 